their psychotherapy Assessment of Clinical Expertise. The trainee particularly would find it useful because it has excellent, up-todate, succinct and clear summaries of theories of cognition, learning and emotion - not what I had expected from the title but relevant to understanding formulation models. What I had expected was a comparison of different ways of formulating cases but instead, more usefully, a straightforward approach is described to developing a problem list, mechanisms and precipitants, and a paragraph summarising the case. Then a series of examples are provided illustrating this, as well as advice on how to use formulation to guide treatment goals and decision-making.

In the process, strategies and techniques are indicated that have since sent me off looking for more detail to use in practice, for example 'caring days' in couple therapy whereby the couple acts 'as if' they cared for each other (it could be usefully adapted for some fraught manager-clinician partnerships). This example appears in a chapter on the therapeutic relationship - again not quite what I had expected in a book on formulation but apposite and reinforcing the importance and ways of developing a working alliance, especially when obstacles get in the way. Any psychological intervention and certainly cognitive-behavioural therapy (CBT) depends on such a firm foundation on which more specific techniques can be built.

The acronym EST gets regular mention but it is not referring to Erhard Seminars Training that strove infamously in the 1970s to allow participants to achieve, in a very brief time (60 hours in two weekends), a sense of personal transformation and enhanced power, but to empirically supported therapies. Its detractors might say that CBT tries to achieve the same but with fewer hours spread thinly over a few months. However, this text claims less - more about coping more effectively - and cites the existing empirical support (evidence).

The case formulation approach seems particularly useful for those patients who do not fit into single DSM or ICD boxes but straddle them - with mental or physical problems - or get lost somewhere inside the vast expanses of 'depressive illness' or 'schizophrenia'. These are those patients we see, as psychiatrists, every day and for that reason one click might just be worth making.

David Kingdon University of Southampton, Royal South Hants Hospital, Brintons Terrace, Southampton SO14 OYG.

doi: 10.1192/bjp.bp.108.062000

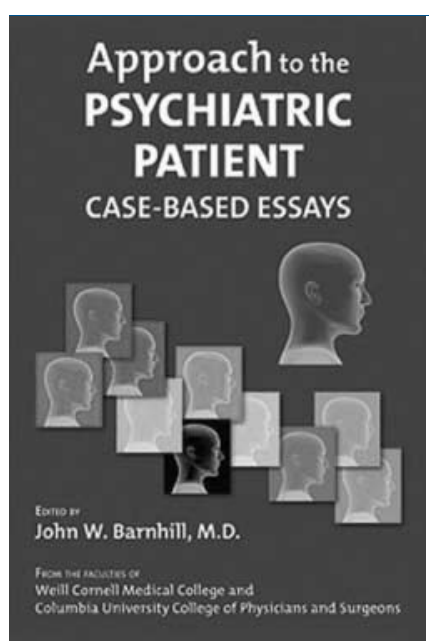

\section{Approach to the Psychiatric Patient. Case-Based Essays}

Edited by John W. Barnhill American Psychiatric Publishing. 2009. US $\$ 62.00$ (pb). 565pp. ISBN: 9781585623006

Although our knowledge of psychiatry has advanced to a large extent, we still know too little and a lot remains to be discovered.
The more we find out, the more we are aware of our limitations and the promises that each discipline holds. This book emphasises the recognition of the usefulness of multiple approaches, presents recent advances to our knowledge base and highlights controversies. It explicitly addresses the multidisciplinary approach and emphasises the importance of coordination and integration between different disciplines.

The book is structured around 10 cases, each followed by 10 to 14 relevant essays. The cases, drawn from clinical experience, are chosen to bring out the complexity and diversity of individuals seen in the clinical setting. They include a wide diagnostic spectrum, from mood instability and schizophrenia to hypomania and geriatric depression. Each essay focuses on one aspect of the case, with authors from various disciplines such as basic science, psychopharmacology, psychiatry, psychology, anthropology, etc. Despite over a hundred contributors, the essays are of consistently excellent quality. An expert provides an overview for each chapter, at the end of which the main points are summarised.

The essays discuss approaches to diagnosis and treatment from the perspective of various disciplines. For instance, the case of double depression is followed by a discussion on biological and cultural factors in the aetiology of depression, suicide, psychodynamic formulation, interviewing depressed patients, the neurobiology of stress, in-patient psychiatry and treatment aspects with regard to psychopharmacology, pharmacogenomics, neuromodulation, supportive psychotherapy and couple therapy. This closely resembles the multidisciplinary working - and the issues that this brings up - in day-to-day clinical practice.

The book mirrors clinical practice also in the way in which the clinician approaches patients, asks questions and finds answers, as well as sometimes accepting uncertainty. All essays may not be relevant to every reader and one may start with those that one finds interesting. This book complements the standard textbook and is not intended to replace it. It is likely to appeal to a wide range of professionals such as experienced psychiatrists, trainees, psychologists and colleagues from nursing and social care backgrounds.

Amit Nulkar Northumberland, Tyne and Wear NHS Trust, Fairnington Centre, Corbridge Road, Hexam NE46 1QJ, UK. Email: amit.nulkar@ntw.nhs.uk

doi: 10.1192/bjp.bp.108.062067

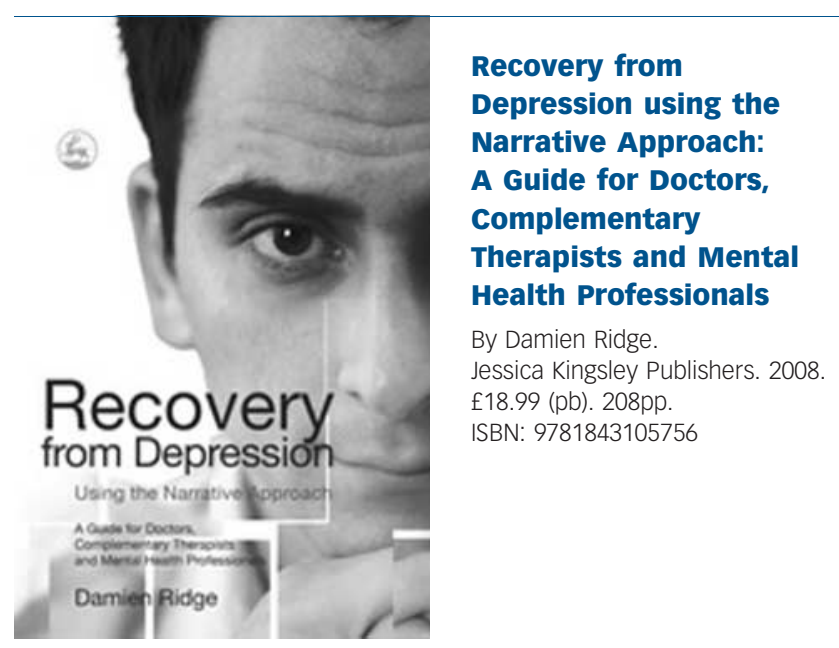

This book presents a piece of narrative research into patients' experiences of depression, recovery and treatments. Ridge carried out in-depth, open-ended interviews on 38 people with a history 